\title{
La médecine basée sur les données probantes ou médecine fondée sur des niveaux de preuve : de la pratique à l'enseignement
}

Bernard GAY *, Marie Dominique BEAULIEU * *

\section{Messages clés}

- Le concept anglo-saxon d'evidence-based medicine, traduit par médecine basée sur les données probantes ou médeaine fondée sur des niveaux de preuve, proposeun nouveau modèe del'exercice médical. • II repose sur la synthèse des trois composantes de la décision (données actuelles de la science, contexte de soins et choix du patient) qui détermine la compétence clinique du médecin. - Sa mise en cauvre dans la pratique ne se réduit pas à l'application systématique des connaissances scientifiques val idées mais intègre dans la démarche décisionnelle les facteurs liés à la situation clinique et à l'opinion du patient. - Ce nouveau paradigme apporte une contributi on aux di fférentes étapes du raisonnement clinique, à la résolution des problèmes complexes et par là même à la structuration des activités d'enseignement et d'apprentissage II et cohé rent avec une perspective socio-cognitivise et suggère fortement la contextualisation des apprentissages • Le recours au concept de médecine basée sur les données probantes dans la formation médicale implique quelques conditions préalables: produi re des données pertinentes et adaptées aux différentsni veaux de soins, intégrer les enseignements dela médeci ne basée sur les données probantes à tous les contextes d'enseignement clinique, assurer la formation des enseignants, fournir aux milieux d'apprentissage des accès aux ressources scientifiques

\section{Mots dés}

evidencebased medicine ; EBM ; médecine basée sur les données probantes ; médecine fondée sur des niveaux de preuve; raisonnement clinique; décision médicale ; contextual isation ; enseignement ; apprentissage

\section{Key Messages}

- The concept of evidence based medicine provides a new model for clinical practice $\bullet$ It lies on the synthesis of the three components of decision making (best scientific evidence, dinical state and patient's preferences) which determines practitioner's clinical competence - Its setting into daily work is not only a systematic application of validated scientific knowledge but integrates in the decision making process the factors related to the clinical stuation and patient opinion. - This new paradigm contributesto the different stages of clinical reasoning, to the resolution of complex problems and consequently to the learning process It is coherent with a socio-cognitivist point of view which suggests a contextualisation of learning. - Facilitating its introduction in medical education implies some prerequistes to produce relevant data, adapted to the different level sof care, to integrate evidence based education into all the contexts of clinical teaching, to ensure the training of the teachers, to provide to the training settings the access to scientific resources

\section{Key words}

evidence-based medicine; EBM ; clinical reasoning ; decision making ; contextualisation; teaching ; learning

PédagogieM édicale 2004 ; 5 : : 171-183

* Département de médecine générale, Université Victor Segalen Bordeaux 2, France.

* * Département de médecine familiale de l'Université de Montréal et Centre de recherche du Centre hospitalier de l'Université de Montréal, Montréal, Québec, Canada

Correspondance: Bernard Gay - Département de médecine générale- Université Victor Segalen Bordeaux 2 - 146 rue Léo-Saignat 33076 Bordeaux Cedex - France - Téléphone : (33) 556629209 - Fax : (33) 556769075 - Mailto:bgay@wanadoo.fr 


\section{Références}

\section{Introduction}

Le concept anglo-saxon d'evidence-based medicine (EBM) pénètre le monde francophone et les propositions de traductions en français sont nombreuses : médecine fondée sur des preuves, médecine fondée sur des niveaux de preuves; médecine fondée sur les faits prouvés; médecine factuelle; médecine basée sur les données probantes.

La difficultévient de la traduction de «evidence» qui peut signifier preuve au sens juridique du terme, mais aussi tout élément servant à fonder un jugement ou une décision. Les résultats d'un essai randomisé sont une « evidence ", I'avis d'un groupe d'experts aussi, de même que l'opinion du patient, mais ces « evidences » n'ont pas la même valeur.

Au Canada, le Forum national sur la santé a proposé comme un des défis pour les prochaines années dans le système de santé canadien de créer une culture de prise de décision fondée sur les données probantes', entérinant par là même une traduction officielle. En France, il n'y a pas de proposition consensuelle mais l'usage semble consacrer le terme de médecine fondée sur des niveaux de preuve. Sans entrer dans une analyse sémantique, cette appellation donne l'impression que toute l'affaire repose uniquement sur l'appréciation des niveaux de preuve scientifique, alors que ce n'est pas le cas. Le mot probant va au delà des niveaux de preuves et intègre les questions de transférabilité aux différents contextes et de jugement sur l'ensemble des données. Le terme EBM est utilisépar facilité parce qu'il est concis, mais les tentatives d'acronymefrancophonen'ont pas abouti, y compris dans ce journal où coexistent M BP (médecine baséé sur les preuves) ${ }^{2}$ et M PA (médecine preuves à l'appui) . $^{3}$.

Alors qu'il était à l'origine destiné à l'enseignement, le concept de médecine basée sur les données probantes a été surtout utilisé pour orienter une réflexion sur les pratiques : la question se pose de son intégration dans l'enseignement médical actuel. Après avoir décrit ce modèle conceptuel explicite pour la pratique, sa contribution au raisonnement clinique et à la structuration des apprentissages sera développée, ce qui permettra d'identifier les enjeux de son utilisation dans l'enseignement.

Enfin, bien quele concept ait étédéveloppé par des méde cins, il a été depuis repris par plusieurs professions du domaine de la santé : evidencebased nuring, evidence based management, etc. D ans tous les cas, le concept est le même et plusieurs des propositions qui seront avancées sont applicables à d'autres disciplines que la médecine. Pour des fins de simplification et parce qu'elle est plus près de la réalité du concept, la formulation médecine fondée sur les données probantes sera utilisée dans ce texte.

\section{Définition}

Un nouveau paradigme est proposé dans les années 1990 : «La pratique fondée sur les données probantes di minuel'emphase donnée à l'intuition, à l'expérience dinique non systé matique et à l'argumentation physopathologique comme bases suffisantes à la prise de décision dinique et insiste sur l'examen des données issues de la recherche dinique " ". Dans cette approche, la décision s'appuie sur des faits et non sur des opinions ou des sentiments. Les données de qualiténe reposent plus sur la physiopathologie ou sur l'expérience, mais répondent aux critères de l'épidémiologie clinique.

Une approche décisionnelle exclusivement intuitive comporte en effet des risques aux conséquences délétères : prendreses opinions pour desfaits établis et croireque son expérience sur un cas isolé a la même val eur que les résultats d'une étude méthodologiquement correcte ; prendre son ignorance pour les réelles limites de la connaissance médicale et ne pas rechercher de données objectives sur le problème.

$M$ ais cette proposition de décision fondée sur les preuves suscite de nombreuses réactions négatives car elle est perçue comme réductrice. En effet, elle semble donner très peu d'importance à l'expérience clinique, considérée jusqu'alors comme un des fondements de l'expertise médicale. Aussi, la définition proposée en 1996 par Sackett précise la démarche et y intègre le patient : «U tilisation consciencieuse, explicite et judicieuse des mélleures données actuelles de la recherche clinique dans la prise en charge personnalisée de chaque patient $»^{5}$. En 2000, Sackett propose une nouvelle définition qui insiste sur la compétence clinique du médecin : «Intégration desmeilleures données dela recherche à la compétence clinique du soignant et aux valeurs du patient ${ }^{6}$.

Cetteévolution de la définition traduit le souci de pondé rer les données actuelles de la science dans un processus de décision qui tienne compte aussi du patient et du soignant dans le contexte de soins. La médecine basée sur les données probantes devient une méthode de formalisation de la décision, opérationnelle dans l'exercice quotidien. 


\section{Un modèle conceptuel explicite pour la pratique \\ Une démarche opérationnelle}

La pratique d'une médecine basée sur les données probantes repose sur les trois composantes de la décision : 1) les circonstances de soins et la situation clinique; 2) les préférences, le comportement et les choix du patient ; 3) les données actuelles de la science apportées par la recherche clinique, avec leurs niveaux de preuve.
Ce modèle peut être schématisé dans une représentation graphique (figure 1) qui fait apparaître la décision à l'intersection des cercles illustrant respectivement ces trois composantes. $\mathrm{D}$ ans cette perspective, la compétence clinique réside dans la capacité du médecin à faire la synthèse de ces trois facteurs afin de prendre la décision la plus adaptée. Cette démarche est utilisée quotidienne ment par la plupart des médecins, de façon souvent empirique et automatique. En effet, les situations courantes se prêtent bien à cette approche qui permet une

\section{Figure 1 : Représentation graphique de l' Evidence-based medicine d'après $\mathrm{H}_{\text {aynes }}$}

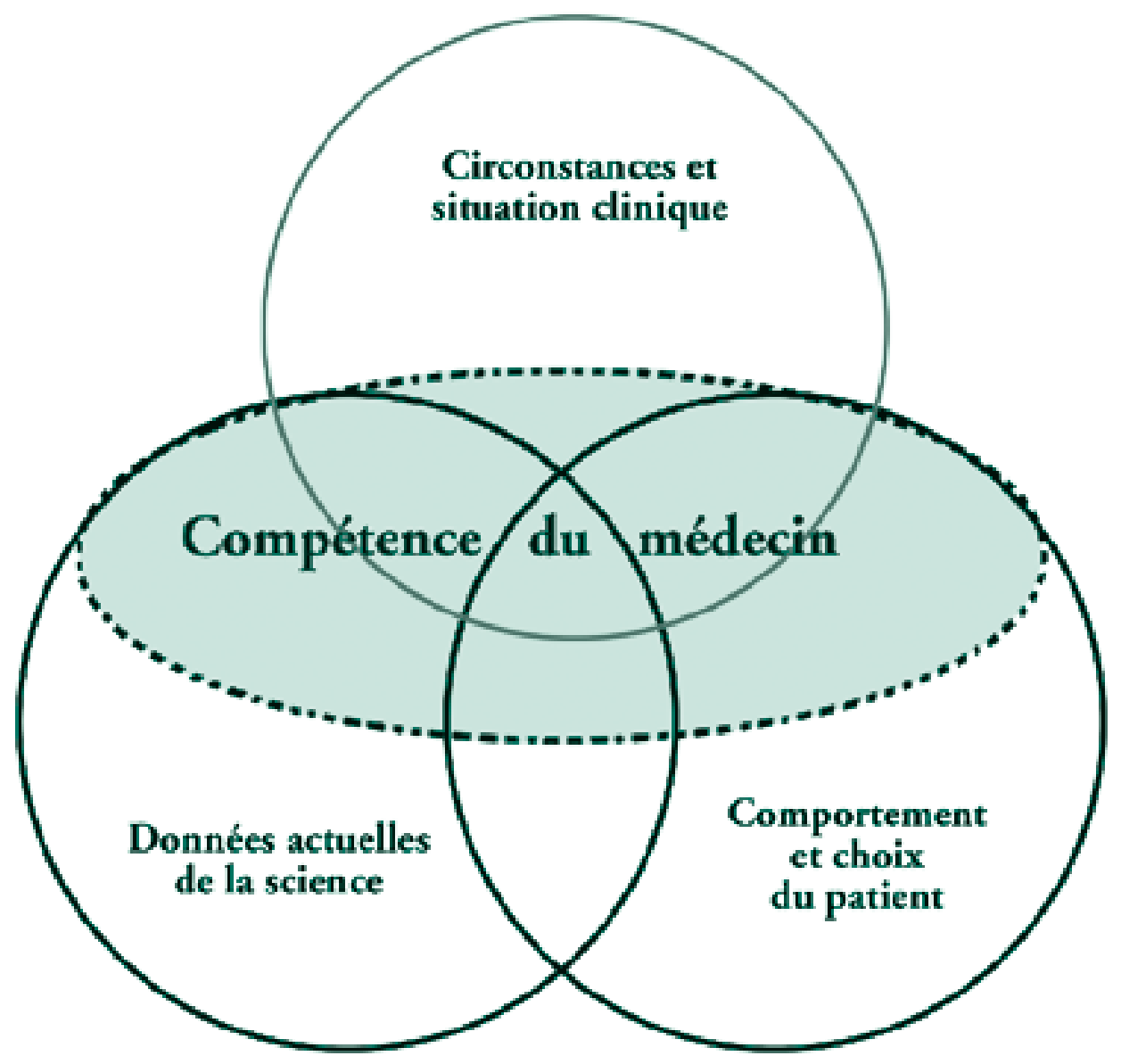




\section{Références}

prise en charge globale des problèmes de santé. La médecine basée sur les données probantes formalise cette démarche, en passant de l'implicite à l'explicite, en rendant visibles les étapes successives d'un processus qui est souvent spontané.

\section{Un processus de formalisation de la décision}

D ans sa description formelle, la démarche décisionnelle basée sur les données probantes se décompose en cinq étapes successives : 1) poser un problème clinique : 2) recueillir les meilleures données di sponibles; 3) analyser la validité de ces données; 4) mettre en adéquation ces données avec les circonstances cliniques ; 5) appliquer ces données de façon personnalisée à chaque cas individuel.

Les trois premières étapes correspondent à l'analyse critique de la littérature, les deux dernières au transfert de connaissances et à leur mise en oeuvre dans la pratique. La compétence du médecin permet d'intégrer et de pondérer les données actuelles de la science en fonction de la situation clinique et des choix du patient. Elle résulte : a) de l'applicabilité des données scientifiques (transposabilité des études expérimentales dans la « vraie vie ») ; b) de l'évaluation du contexte de la situation de soins (gravité, pathologies associées, ressources disponibles, caractéristiques de la discipline) ; c) de l'appréciation du problème du point de vue du patient (représentations, valeurs, information).

La démarche clinique tient compte des trois composantes de la décision, sans négliger l'une ou l'autre:

- l'accès aux données actuelles de la science nécessite une veille documentaire qu'un médecin isolé peut difficilement réaliser. La littérature quaternai re ${ }^{8}$ permet, avec les recommandations pour la pratique clinique, d'accéder à une synthèse argumentée de l'information médicale. La gradation des recommandations ${ }^{9}$ donne le degré de validité en fonction du niveau de preuve ${ }^{10}$ des études qui ont conduit à leur proposition. Le risque d'obsolescence des recommandations, en raison de l'évolution rapide des connaissances et du délai d'élaboration, impose leur actualisation périodique ${ }^{11}$. Entre temps, les revues méthodiques du type de celles élaborées par la Cochrane Collaboration ${ }^{12}$ peuvent apporter une information plus récente. $D$ ans tous les cas, les résistances individuelles au changement ${ }^{13}$ limitent I'utilisation des recommandations et freinent leur mise en œuvre.
- Les circonstances et la situation cliniques sont appré ciées au cas par cas. Le niveau d'intervention (soins primaires, secondaires ou tertiaires), les caractéristiques de l'exercice, le stade d'évolution de la maladie, le cadre géographique de la pratique (cabinet ou domicile, milieu urbain ou rural), l'état clinique du patient, les ressources disponibles, conditionnent la réponse au problème. L'influence du système de distribution des soins ne peut pas être oubliée avec les contraintes que celui-ci impose à l'exercice médical.

- Les comportements spécifiques et les préférences du patient dépendent de sa personnalité, de son histoire personnelle et familiale, de ses représentations de la santé et de la maladie, de ses valeurs socio-culturelles, de l'information médicale médiatisée. La loi sur les droits des malades en France ${ }^{14}$ confirme la nécessité d'informer le patient, de recueillir son opinion et d'intégrer son avis dans la décision. II nes'agit pas de se plier aux exigences du patient mais de rechercher ce qu'il est prêt et capable d'accepter. Ce partage de la décision est une évolution significative de la pratique médicale. II nécessite de fournir aux patients des aides à la décision ${ }^{15}$ qui lui permettent de faire des choix éclai rés parmi les différentes options possibles.

\section{Une mise en œuvre flexible}

C e modèle est séduisant mais peut paraitre un peu rigide. Le risque de normalisation des pratiques est souvent avancé par les opposants qui fustigent la prééminence biomédicale que ce modèle implique à leurs yeux. En effet, pour beaucoup de médecins, la médecine basée sur les faits probants se réduit à une application systé matique et sans nuance des données acquises de la science, dans une perspective positiviste. Cette dérive, qu'illustre d'ailleurs bien la traduction d'EBM utilisée en France, décrédibilise l'idée de médecine basée sur les données probantes. En réalité, la définition précise clairement la place du contexte clinique et des choix du patient dans la décision. La description rend bien compte de la nécessaire personnalisation des soins selon l'importance de l'un ou de l'autre des éléments de la décision dans chaque situation. Cette approche plus éclectique est cohérente avec une perspective constructiviste des problèmes de santé. De plus, la variabilité potentielle de la proportion respective des trois cercles du schéma permet de donner la flexibilité nécessaireà ce modèle. II est des situations dans lesquelles les données 
actuelles de la science sont rares ou contradictoires et laissent une place encore plus grande aux choix du patient. II est des situations dans lesquelles le contexte clinique est tellement particulier qu'il prend le pas sur les deux autres facteurs.

\section{Une démarche pédagogique à partir de la médecine basée sur les données probantes}

Pour certains formateurs, l'enseignement de la médecine basée sur les données probantes se résume à l'enseignement de l'analyse critique de la littérature médicale: maitriser les concepts de l'épidémiologie clinique et les appliquer à la lecture d'articles scientifiques, souvent dans le cadre de clubs de lecture. La proposition formulée dans ce texte offre une vision beaucoup plus large de la contribution du concept de médecine basée sur les données probantes à la formation des professionnels de la santé : faciliter l'acquisition du raisonnement clinique et la prise de décision dans des situations complexes. En conséquence, ce concept est de nature à contribuer à la structuration de la démarche pédagogique menant à la maîtrise des compétences essentielles au clinicien.

\section{Contribution à la modélisation du raisonnement clinique}

La médecine basée sur les données probantes, c'est d'abord une attitude, une relation aux connaissances biomédicales : ce n'est pas une « recette » que le médecin utilise ou non. Putman parle d'« evidence mindness $»^{16}$, Junod évoque " un état d'esprit plus qu'une méthode $\aleph^{17}$. L'évaluation et l'utilisation judicieuse des connaissances biomédicales nécessitent une habileté dont aucun médecin ne peut prétendre se passer. Elle supporte ce qui est à la base même de l'expertise médicale, à savoir le raisonnement clinique, auxquels s'ajoutent la capacité de recueillir l'histoire clinique du patient et les habiletés cliniques (sémiologie, examen physique).

En effet, les connaissances biomédicales et l'expérience (qui bâtit ce que Schön ${ }^{18}$ et d'autres appellent la metaconnaissance) sont à la base du raisonnement clinique. Le développement de l'expertise est largement une question de réorganisation des connaissances et des processus cognitifs de performance de tâches ${ }^{19}$.

Les connaissances biomédicales et l'expérience sont mobilisées pour la génération d'hypothèses qui initient le processus hypothético-déductif, en une étape spontanée et automatique. C'est une démarche classiquement utilisée dans le raisonnement médical ${ }^{20}$, qui fait appel aux connaissances, elles mêmes organisées selon divers modèles, sous forme de représentations cognitives complexes comme par exemple les «scripts » clinique ${ }^{21,22}$ ou les réseaux sémantique ${ }^{23}$. Les différentes couches de connaissances sont basées sur des associations, en une série d'attributs, chacun recevant une probabilité d'occurrence plus ou moins élevée selon un contexte spécifique. Par exemple, le «script » d'une angine d'origine streptococcique est constitué d'une série d'attributs comme la présence d'hyperthermie, d'adénopathies cervicales, d'exsudat, etc., auxquels une probabilité d'occurrence est attribuée (une sorte de valeur « défaut »). Les valeurs défauts sont issues de l'expérience mais aussi de la recherche clinique sur la valeur prédictive des signes et des symptômes.

Le modèle de Barrows ${ }^{24}$ permet d'illustrer cette démarche en montrant que la médecine basée sur les données probantes peut s'appliquer à chacune des étapes du processus de raisonnement (figure2).

Les connaissances issues de la recherche clinique et épidémiologique, contributives aux représentations cognitives mobilisables lors du processus de résolution de problème, concernent des domaines variés. Q uelques uns peuvent être évoqués à titre d'exemple :

- Perception, miseen contexte: prévalence en fonction des caractéristiques sociodémographiques (âge, sexe, culture, pays, etc.).

- Analyse : confrontation des éléments de l'histoire et de l'examen à un ensemble de «scripts » afin d'établir les hypothèses les plus plausibles.

- Stratégie de questionnement : valeur prédictive des signes et symptômes.

- Choix des tests: définition de la normalité; sensibilité, valeurs prédictives selon différentes prévalences.

- Traitement : efficacité, risques, conséquences des différentes options, conséquences de la non-intervention (histoire naturelle).

- D iagnostic: probabilité de maladie selon les résultats.

Les étapes nécessaires à un raisonnement clinique de qualité sont rappelées par $\mathrm{N}$ endaz ${ }^{25}$. La phase initiale de repré sentation du problème permet d'activer des connaissances organisées et élaborées, ce qui génère des hypothèses dia gnostiques pertinentes. L'interprétation des données et 


\section{Références}

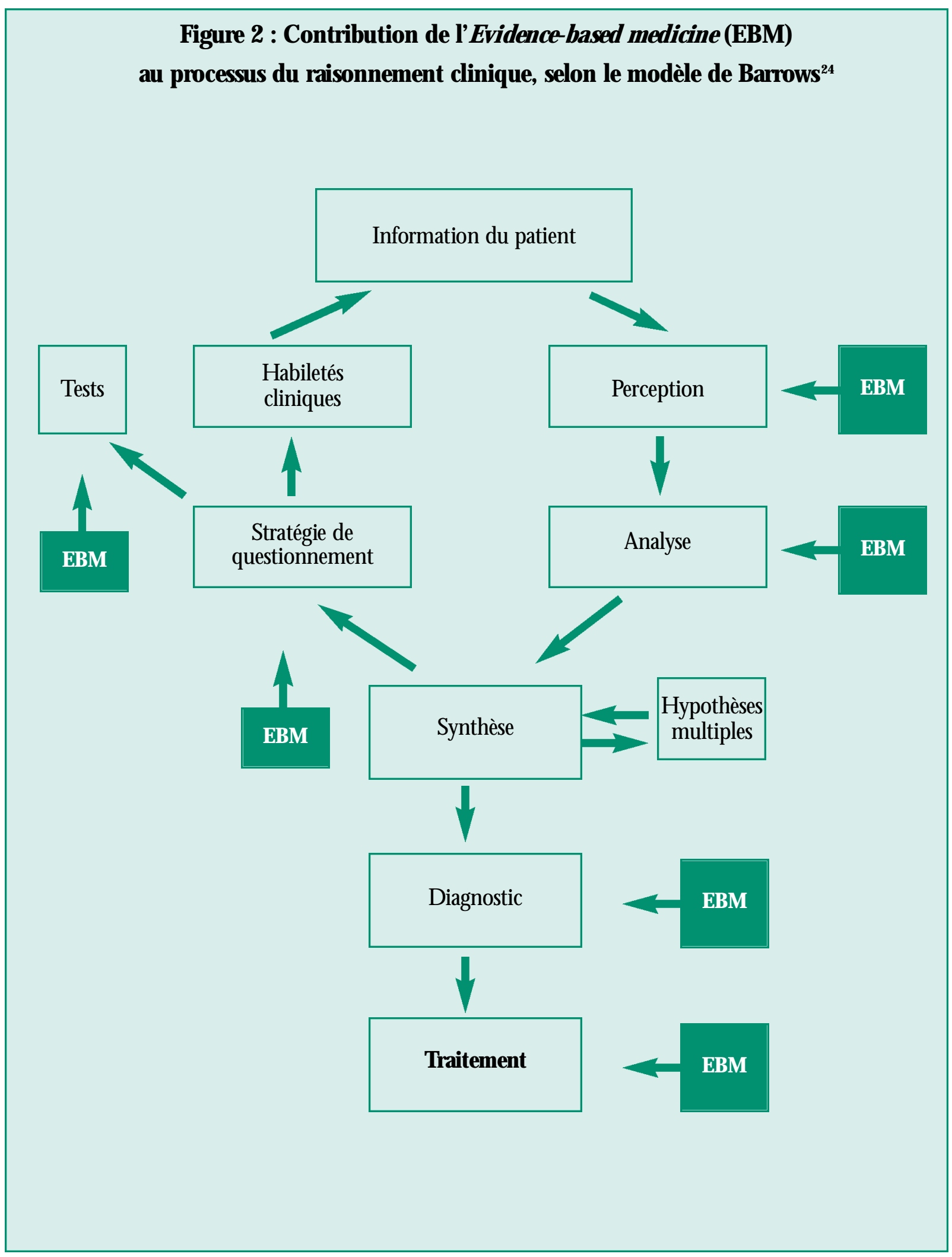


l'évaluation des hypothèses servent de guide pour obtenir des données supplémentaires qui viennent modifier la représentation initiale jusqu'à l'obtention d'un diagnostic clinique.

Le recours explicite aux connai ssances biomédicales formelles dans le processus de raisonnement clinique diminue avec l'expertise ${ }^{26}$. Chez les cliniciens d'expérience, certains travaux suggèrent qu'après six mois, une nouvelle information biomédicale était intégrée dans le réseau de connaissances et mobilisable de façon automatisée ${ }^{16}$.

Les connaissances biomédicales restent néanmoins pré sentes et accessibles2 : elles placent les contraintes sur les valeurs acceptables des scripts; elles alertent le clinicien quand une découverte ne correspond pas à ce qui était attendu ; elles sont aussi utilisées explicitement dans des situations où les scripts ne sont pas élaborés pour géné rer des hypothèses. Les schémas opératoires qui fondent la compétence médicale associent d'ailleurs les connaissances biomédicales formelles et les connaissances issues de l'expérience au sein de réseaux cognitifs complexes grâce à de riches interconnexions. Un raisonnement clinique basé sur des connaissances erronées ou inappropriées ne peut pas être optimal. La validité des sources est donc une condition essentielle bien qu'elle ne soit pas seule garante d'un raisonnement clinique adéquat.

Ces données suggèrent la nécessité que, lors des activités d'enseignement et d'apprentissage du raisonnement clinique, la démarche pédagogique prenne en compte I'organisation des connaissances et le processus cognitif. L'enseignant doit développer des interventions facilitantes appropriées:

- présenter des connaissances rigoureuses aux étudiants, comme bases de leur savoir biomédical : questionner les critères de rigueur, développer I'« evidence mindness » ; - donner les clés qui permettront aux étudiants d'évaluer les connaissances produites pour mettre à jour leur «base de données 》 (leurs connaissances antérieures), au fur et à mesure (en se rappelant que la qualité des connaissances ne peut s'évaluer que dans une mise en contexte, car l'évaluation des critères scientifiques d'une étude est directement liée à la question clinique à laquelle elle tente de répondre) ;

- développer le réflexe de remise en question des connaissances sur lesquelles le médecin base son raisonnement. Cette démarche fait partie du processus de raisonnement comme les autres réflexes (celui de revoir les hypothèses, de se questionner sur son état de réceptivi- té, sur ses propres biais personnel, etc.). Elle relève de la métacognition (évaluation de ses propres connaissances et processus cognitifs en situation d'action).

Cette contribution du concept de médecine basée sur les données probantes au raisonnement cliniqueest pertinente. M ais plusieurs obstacles en limitent l'applicabilitéez :

- absence de données validées dans certains domaines (soins primaires vs soins secondaires ou tertiaires) et pour certains sous-groupes de patients ;

- connaissances parfois décalées du contexte clinique : la production des données actuelles de la science fait appel à des études le plus souvent expérimentales, produites dans des contextes cliniques contrôlés ;

- recherche des données et analyse critique très chronophages à la fois pour les étudiants et les professeurs.

De plus, l'approche critique des données scientifiques, si elle est trop prématurément mise en avant, peut déstabiliser les étudiants qui, au cours des premières années de leur formation, ont tout à apprendre. U ne grande partie des connaissances à maîtriser au début des études médicales est de nature fondamentale et physiopathologique. Enfin, la transposabilité des données expérimentales n'est pas toujours possible : les patients qui consentent à participer aux essais cliniques sont différents de ceux qui ne consentent pas; les patients polypathologiques sont en général exclus; le contexte de I'intervention clinique est souvent spécifique ; l'effet $\mathrm{H}$ awthorne ${ }^{28}$ (tendance des investigateurs à améliorer leur performance quand ils savent qu'ils sont observés) peut perturber l'analyse.

Ces limites sont toutes réelles mais, pour la plupart, elles font parties des limites inhérentes à la production et à l'utilisation des connaissances dans le domaine de la santé. II est important d'initier les étudiants à ces réalités et de leur donner les outils pour y faire face. Le choix des références à proposer doit favoriser les synthèses de l'information (littérature quaternaire) qui font gagner du temps et représentent le type de littérature la plus consultée dans la pratique. II est cependant indispensable que les étudiants comprennent le mode d'élaboration et les limites propres de cette littérature médicale.

\section{Contribution à la modélisation du processus de résolution des problèmes complexes}

Le médecin doit être en mesure d'offrir des services 


\section{Références}

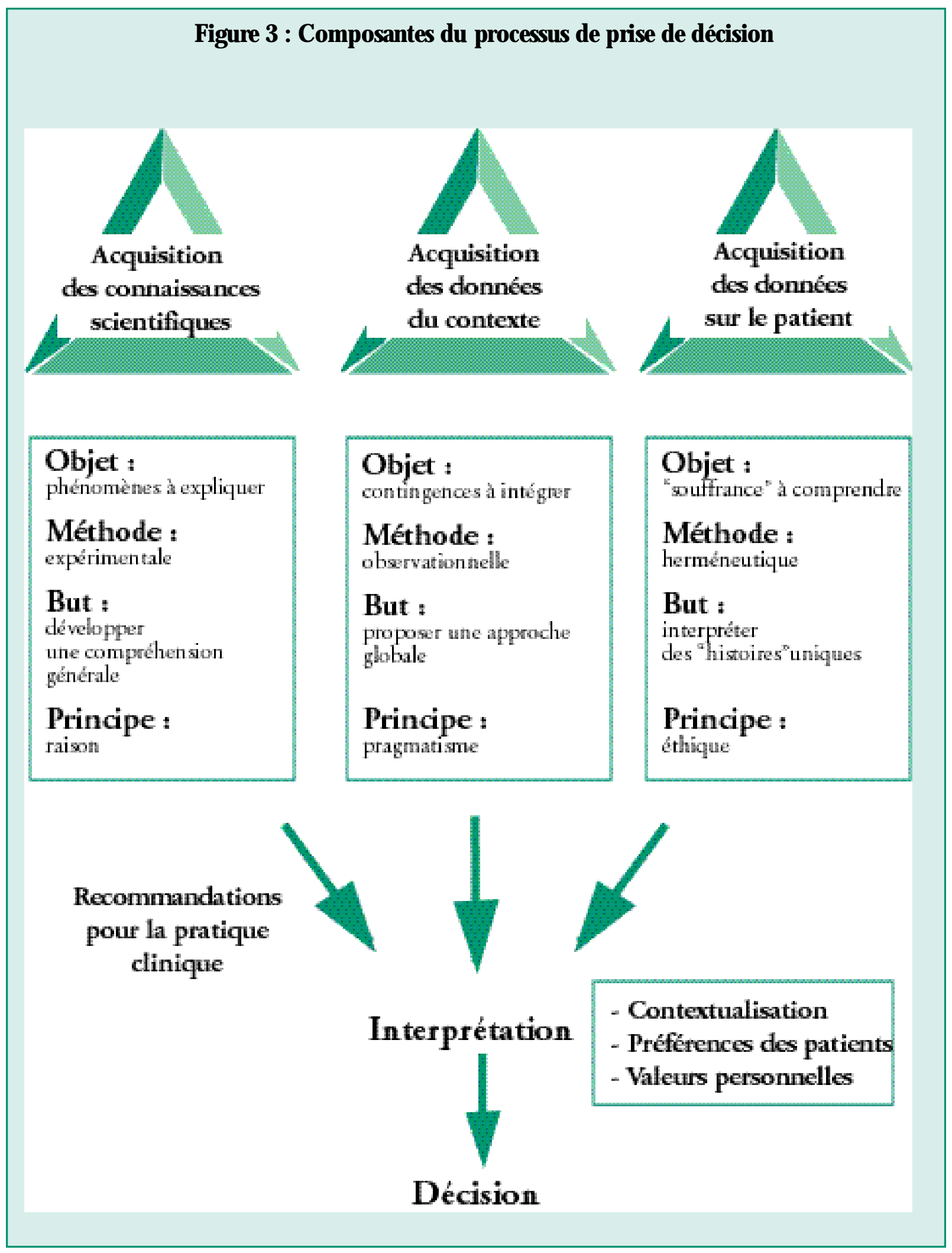


judicieux et réfléchis, dans des situations toujours uniques, complexes et floues, où il est fort probablequ'il soit confronté à des conflits de valeur et à des problèmes éthiques ${ }^{18,29,30}$. Les données actuelles de la science, seules, ne permettent pas de résoudre de tels problèmes complexes. II ne suffit pas de faire une analyse de la littérature pour être dans une démarche basée sur les données probantes (club de lecture, revue bibliographique) : le risque est d'isoler les connaissances du contexte clinique.

En partant dela représentation de la prise de décision proposée par Beaulieu ${ }^{31}$ et en y intégrant les données du contexte, il est possible de développer un processus de prise de décision qui découle directement du concept de médecine basée sur les données probantes (figure 3).

Cette approche tridimensionnellen'est pas sans rappeler le modèlebiopsychosocial d'Engel ${ }^{32}$ qui prend en compte les aspects biomédicaux, psychocomportementaux et socio environnementaux. Elle facilite la gettion de l'incertitude et de la complexité et permet une prise de décision même en l'absence de données validées.

\section{Contribution à la structuration des activités d'enseignement et d'apprentissage}

$H$ istoriquement, la perspective de la médecine basée sur les données probante avait comme préoccupation premiè re l'enseignement. Telle qu'elles'est développée et codifiée ces dernières années, en tant que paradigme pour la pratiquemédicale, ellene postule pas d'orientation théorique particulière concernant l'enseignement ou l'apprentissage. En proposant un cadre conceptuel général de l'action médicale et du processus de résolution de problème en médecine, elle offre cependant un modèle contributif pour illustrer auprès des étudiants la finalité de leurs apprentissages et pour aider les enseignants à concevoir leurs interventions. En mettent l'accent sur le processus de résolution de problèmes, cette perspective se révèle par ailleurs fortement convergente avec la perspective cognitiviste de l'apprentissage et avec le courant de l'enseigne ment contextualisé

Transposées dans l'enseignement, les étapes de la pratique médicale suggérées par la médecine basée sur les données probantes sont utilisables de manière cohérente avec les deux orientations précédentes:

1. Amener l'étudiant à poser une question clinique.
2. Faciliter la recherche des meilleures données disponibles.

3. Stimuler l'analyse des données

4. Encourager la mise en adéquation des données avec les circonsances di niques.

5. Favoriser l'application personnalisée de ces données à chaque cas individuel.

D ans cette dynamique, trois questions se posent à l'enseignant : Comment préparer l'étudiant aux situations dans lesquel les il n'y a pas de données val idées ? Faut-il dire aux étudiants que la charge de la preuve peut résider dans la démonstration del'efficacité ou del'inefficacité? Q ue dire aux étudiants sur les circonstances dans lesquelles il faut rechercher des données validées pour aider la décision diagnostique ou thérapeutique ${ }^{33}$ ?

L'accèsà l'information n'est pas la seule façon d'amener les étudiants à entrer dans une médecine basée sur les données probantes. Les situations d'incertitude ou de complexité requièrent une approche particulière que les données actuelles de la science ne peuvent pas toujours régler. L'apprentissage doit faire le lien entre la science et la pratique et associer à la connaissance formelle, issue des résultats de la recherche, les savoirs pratiques, acquis dans des situations authentiques, au contact des patients dans un contexte de soins donné34. Les compétences développées ne concernent pas seulement l'expertise dinique, mais aussi l'empathie, l'aptitude à la communication, le développement personnel, permettant de proposer un modèle pour la sélection, la formation et la progression des éudiants, en particulier en médecine général $e^{35}$. L'objectif est de développer une manière de penser sappuyant sur les trois éléments de la décision, sans privilégier systématiquement et a priori l'un ou l'autre. II s'agit d'un apprentissage contextualisé de compétences globales organisées en schémas opératoires, de l'application d'une démarche réflexive en situation authentique : "La compétence n'est pas le produit d'une accumulation des savoirs : elle est avant tout une disposition à agir qui sacquiert dans l'action » (M invielle ${ }^{36}$.

La difficulté est dans le changement de paradigme éducationnel qui est ainsi, de fait, proposé. La médecine basée sur les données probantes est sous-utilisée dans I'enseigne ment médical car elle procède implicitement d'une «certaine conception de l'enseignement où le contenu cognitif n'est pas la seule finalité mais où la capacité à identifier l'information pertinente et d'en établir la validité compte tout autant $»^{7}$. 


\section{Références}

\section{Des enjeux pour le développement de la médecine basée sur les données probantes dans l'enseignement}

L'apprentissage a pour finalité l'acquisition des compé tences nécessaires à la prise en charge des problèmes de santé dans le champ d'activité concerné. La mise en situation authentique permet le développement de la performance nécessaire. II peut paraitre difficile d'appliquer la médecine basée sur les données probantes à tous les contextes d'enseignement. Les conditions favorables ne sont pas toujours réunies pour cette nouvelle approche pédagogique et quelques pré-requis sont indispensables.

\section{Produire des données pertinentes et adaptées aux différents niveaux de soins}

Le premier enjeu concerne la production des données scientifiques et $n$ 'est pas spécifique à l'enseignement. Les limites de la médecine basée sur les données probantes sont inhérentes aux limites imposées par la démarche scientifique dans le domaine biomédical :

- contrôle des situations expérimentales;

- choix d'objets de recherche qui peuvent être pertinents sur le plan de l'avancement des connaissances biomédicales mais pas de façon évidente sur le plan clinique;

- transférabilité entre milieux de soins (en particulier des milieux de soins secondai res ou tertiaires vers les milieux de soins primaires) ;

- mise en contexte des données ;

- inférence à des individus.

Ces limites sont liées notamment à la très large prééminence du paradigme positiviste en recherche bioclinique, qui tend à privilégier la validité interne des études (la solidité de la preuve expérimentale) au détriment de sa validité externe, c'est-à-dire de la généralisabilité des résultats ${ }^{37}$. L'important développement actuel des travaux cliniques s'inscrivant dans une perspective différente, celle la recherche qualitative $e^{38,39}$, devrait à terme contribuer à faire admettre une conception plus éclectique des données probantes.

Lemouvement en faveur de la prise de décision clinique fondée sur des données probantes a imposé des pressions aux milieux de recherche qui sont devenus plus sensibles aux considérations cliniques. Par exemple, une objection classique des généralistes est l'absence de données issues des soins primaires permettant d'étayer une décision. En fait, une étude rétrospective sur 122 consultations montrait que $81 \%$ des décisions disposaient d'essais cliniques randomisés ou de données cliniques convaincantes publiées dans la littérature ${ }^{40}$.

Enfin, il ne faut pas non plus négliger le fait que le discours sur les données probantes a aussi été repris par les différentes industries de la santé (pharmaceutique, technologique, etc.), ainsi que par les gouvernements, et qu'il est de ce fait au centre des arbitrages sur les coûts et les choix politiques en matière de santé. $D$ ece fait, les données scientifiques ne sont pas exclues des enjeux de pouvoir, ce qui rend certains sceptiques sur leur valeur. Sans laisser le cynisme envahir le débat, il est clair qu'il sagit d'une limite importante dont les étudiants doivent apprendre à tenir compte.

\section{Intégrer les enseignements de la médecine basée sur les données probantes à tous les contextes d'enseigne- ment clinique}

$D$ ans la mesure où la pratique de la médecine fondée sur les données probantes est avant tout une attitude, une approche des connaissances scientifiques et de leur application dans le domaine clinique, il est important d'en intégrer les concepts dans les différentes activités d'apprentissage. II faut voir plus large que l'enseignement théorique de l'épidémiologie clinique dans le cadre de clubs de lecture. Les cinq étapes de la médecine basée sur les données probantes s'appliquent à toutes les formes d'enseignement :

- dans les premiers cycles : approche par problème, cours magistraux ;

- par nature, dans les activités d'apprentissage du raisonnement clinique, que les formats en soient codifiés (ARC) ou non ;

- Iors des activités de supervision clinique, directe (au lit du malade) ou indirecte, et lors des discussion de cas ;

- en formation médicale continue;

- en situation d'interaction avec les collègues et les consultants.

\section{Assurer la formation des enseignants}

La tentation à laquelle il faut résister est de déléguer un enseignant qui devient un «champion » de la médecine 
basée sur les données probantes, alors que ces concepts ne sont repris par aucun autre enseignant. Le message serait alors clair pour l'étudiant : conformément aux principes du curriculum cachédi ${ }^{41}$ la médecine basée sur les données probantes ne serait pas utile pour la pratique. D e plus, sans une intégration à la supervision clinique, qui permet une contextualisation et de multiples recontextualisations, facteurs de transfert des apprentissages ${ }^{42}$, l'étudiant n'apprend pas les étapes essentielles de la médecine basée sur les données probantes que sont l'analyse de l'applicabilité au contexte clinique spécifique et la prise en compte des éléments contextuels et préférences des patients.

Tous les enseignants doivent être en mesure de reprendre les étapes de la médecine basée sur les données probantes, soit en les rendant explicites dans les enseignements plus formels comme les cours magistraux, les apprentissages par problèmes (APP) et la supervision clinique, soit en les modélisant eux-mêmes dans leurs discussions avec des collègues et avec les patients. D ès lors, il devient important de former l'ensemble du corps professoral en étant conscient que de nombreux cliniciens ont encore des réticences face à cette approche de la pratique.

L'exemple de la Best Evidence Medical Education (BEME) Collaboration ${ }^{43}$ qui propose d'utiliser la meilleure « evidence » disponible dans l'éducation médicale, est à ce titre intéressant. Le développement des connaissances actuelles sur l'efficacité des méthodes pédagogiques, la production de revues systématiques sur le sujet et la promotion d'une culture commune à tous les enseignants, représentent une contribution utile à la formation pédagogique des intervenants.

\section{Fournir aux milieux d'apprentissage des accès aux ressources scientifiques}

Les enseignants ont pour tâche de développer les habiletés de « raisonnement » sur les connaissances scientifiques et d'expliciter ses analogies avec le processus même du raisonnement clinique : comprendre quelles sont les questions à poser aux « données scientifiques » (ou à ceux qui les présentent) pour juger de leur qualité scientifique.

II devient donc essentiel que les milieux cliniques permettent l'accès aux informations scientifiques et aux synthèses. Cette possibilité peut poser problème pour les programmes reposant sur l'assistanat dans des milieux cliniques de la communauté, comme c'est le cas en médecine générale, alors que le modèle de rôley tient une place importante.

Un autre difficulté réside dans l'accès à l'information en situation de soins : comment disposer des données en temps réel ? Par exemple, les règles de prédiction clinique qui quantifient les éléments contribuant à la réponse médicale, sont utiles au chevet du patient ${ }^{44}$.

L'informatisation, en particulier la multiplication des Personal Digital Assistant ou le développement des « reminders », devrait permettre de proposer des ressources documentaires au moment même de la décision.

\section{Conclusion}

La médecine basée sur les données probantes est un concept émergeant dans les pays francophones, qui doit garder l'approche tridimensionnelle de son origine anglo-saxonne. La décision résulte de la synthèse extemporanée des données actuelles de la science, des choix du patient et des circonstances de soins, que la compé tence du médecin permet d'adapter au problème posé. La médecine basée sur les données probantes développe une contribution au raisonnement clinique utilisable dans la pratique comme dans I'enseignement. Le processus de résolution de problèmes complexes et la contextualisation des situations d'apprentissage permettent de proposer un nouveau paradigme éducationnel tenant compte des trois composantes de la décision. C ette démarche est en mesure d'influencer positivement la qualité des enseignements, donc la qualité des pratiques, débouchant in fine sur une amélioration de la qualité des soins. 


\section{Références}

\section{Références}

1. Forum national sur la santé. La santé au Canada : un héritage à faire fructifier. Rapports de synthèse et documents de référence, volume 2. Ottawa : G ouvernement du Canada, 1997.

2. Colin R. M édecine basée sur les preuves et éducation médicale Pédagogie médicale 2001;2 : 69-70.

3. Vadeboncoeur A. Réapprendre à apprendre. Pédagogie médicale $2003 ; 4: 9-10$.

4. EvidenceBased M edicine Working Group. Evidence based medicine. A new approach to teaching the practice of medicine. JAM A $1992 ; 268: 2420-2425$.

5. Sackett D L, Rosenberg W M, Gray M uir JA, HaynesRB, Richardson W. Evidence based medicine: what it is and what it in't. BMJ 1996 ; $312: 71-72$.

6. Sackett DL, Straus SE, Richardson WS, Rosenberg W, Haynes RB. Evidence based medicine how to practice and teach EBM. 2d ed. Edinburgh: Churchill Livingston, 2000.

7. H aynes RB, D evereaux PJ, Guyatt GH . La compétence du clinicien à l'ère de la médecine fondée sur les niveaux de preuve et de la décision partagée avec les patients. EM B Journal (édition française) $2003 ; 34 ; 5-8$.

8. Groupe de Recherche ed d'Action pour la Santé Accessible en ligne sur : http://www. grouperechercheactionsante. com/lectureEBM .htm

9. Harbour R, M iller J. A new system for grading recommendations in evidence based guidelines BMJ 2001 ; $323: 334-336$.

10. Agence $N$ ationale d'Accréditation et d'Evaluation en Santé Guide d'analyse de la littérature et gradation des recommandations. Recommandations pour la pratique dinique. Paris: AN AES, 2000.

11. SchekelleP, EcdesM P, Grimshaw JM , WoolfSH . When should dinical guidelines beupdated? BM J $2001 ; 323$ : 155-157.

12. The Cochrane library. Accessible en ligne sur : http://www.update software.com/cochrane/
13. Freman AC, Sweney K. Why general practitioners do not implement evidence qualitativesudy. BMJ $2001 ; 323: 1-5$.

14. Loi $n^{\circ} 2002-303$ du 4 mars 2002 relative aux droits des malades et à la qualité du système de santé Journal officiel de la République française 5 mars 2002.

15. 0'C onnor A. Des aides pour permettre au patients de prendre des décis ons fondées sur des niveaux de preuve. EBM Journal (édition française) $2002 ; 30: 7-8$.

16. Putman W, Twohig PL, Burge FI, Jackson LA, Cox JL. A qualitative sudy of evidence in primary care: what the practitioners are saying. CM AJ $2002 ; 166: 1525$ 1530.

17. Junod AF. Décision médicale ou la quête de l'explicite Genève: M édecine et hygiène, 2003.

18. Schön D A. The reflective practitioner : H ow professionals think in action. $\mathrm{N}$ ew York : Basic Books, 1983. Traduction française : H eynemand J, Gagnon D. Le praticien réflexif : à la recherche du savoir caché dans l'agir professionnel. Montréal (QC) : Les Editions Logiques, 1994

19. Feltovich PJ. Expertise reorganizing and refining knowledge for use Profess ons Educ Res N otes $1983 ; 4: 5-9$.

20. Elstein AS, Scharz A. Clinical problem solving and diagnosic decision making : selective review of the cognitiveliterature BM J $2002 ; 324$ : 729-732.

21. Schmidt H G, N orman GR, Boshuizen H P. A cognitive perspective on medical expertise : theory and implication. Acad M ed $1990 ; 65$ : 611-621.

22. Charlin B, Tardiff, Boshuizen H PA. Scripts and medical diagnostic knowledge : theory and applications for clinical reasoning. Acad M ed $2000 ; 75$ : 182-190.

23. Bordage G, Lemieux M. Semantic structures and diagnotic thinking of expertsand novices Acad M ed 1991; 66 (9 Suppl) : S70-72.

24. Barrows HS, Pickell GC. Developing dinical solving skills. A guide to more effective diagnosis and treatment. N orton M edical Books, N ew York, 1991, 226p. 
25. Nendaz MR. Comment les médecins rai sonnent-ils ? In : Junod AF (Ed.). D écision médicale ou la quête de l'explicite Genève: M édecine et hygiène, 2003 : 17-31.

26. Boshuizen H P, Schmidt H G. On the role of biomedical knowledge in dinical reasoning by experts, interme diatesand novices Cogn Sci $1992 ; 16$ : 153-184.

27. Straus SE, Finlay A. M CAlister FA. Evidence based medicine a commentary on common criticisms CM AJ 2000;163: 837-841.

28. Effet Hawthorne. Accessible en ligne sur : http://c2.com/cgi/wiki?H awthorneE ffect

29. M CG uire $C$. Sociocultural changes affecting professions and professionals In : Curry L, Wergin JF E al.(eds). Educating professionals, San Fransisco (CA) : JosseyBass, 1993 : 3-16.

30. Harris।B. N ew expectations for professional competence In : Curry L, Wergin JF et al. (eds). Educating professionals. San Fransisco (CA) : Jossey-Bass, 1993 : 17-52.

31. Beaulieu M D, Battisa RN, BlaisR. A propos del'« evidencebased medicine». Ruptures $2001 ; 7: 120-34$.

32. Engd GL. The biopsychosocial model and medical education. N Engl J M ed $1982 ; 306: 802-5$.

33. Welch HG, Lurie JD. Teaching Evidence-Based M edicine: Caveats and Challenges Acad M ed 2000 ; $75: 235-40$.

34. Greenhalgh $T$, Toon P, Russell J, Wong G, Plumb L, $M$ affarlane $F$. Transferability of principles of evidence based medicine to improve educational quality: systematic review and case study of an online course in primary health care. BMJ $2003 ; 326$ : 142-145.

35. Patterson $F$, Ferguson $E$, Lane $P$, Farrel $K, M$ artlew J, Wels A. A competency model for general practice: implications for selection, training, and development. BMJ $2000 ; 50: 188-193$.

36. M invielleY. Q uatre thèses sur la compétence. La lettre de la formation $1993 ; 104: 4-7$.

37. Jouquan J. L'évaluation des pratiques confrontées à l'exigence de la preuve scientifique en médeane dinique. M édecineT hérapeutique2003 ; 9 : 93-100.
38. M alterud K. Q ualitative research : standards, challenges and guidelines Lancet $2001 ; 358$ : 483-488.

39. Giacomini M K. The rocky road : qualitative research as evidence. Evid Based M ed $2001 ; 6$ : 4-6.

40. Gill P, D owell AC, N eal RD, Smith N, Heywood P, Wilson $\mathrm{AE}$. Evidence general practice : a retrospective study of interventions in one training practice. BM J 1996 ; 312 : 819-821.

41. H afferty F, Franks R. The H idden Curriculum, Ethics Teaching, and the Structure of Medical Education. Acad M ed $1994 ; 69$ : 861-871.

42. Tardif J. Le transfert des apprentissages Montréal (QC) : Les Editions Logiques, 1999.

43. Best Evidence Medical Education Collaboration. Accessible en ligne sur : http://www.bemecollaboration.org/

44. M CGinn T, M oore C, HoW. Le rendez-vous des praticiens : les règles de prédiction clinique en pratique EBM Journal (édition française) $2003 ; 35: 8$-9. 